\title{
Aceleración y enriquecimiento curricular para estudiantes con altas capacidades: una perspectiva de la situación en el Perú
}

\author{
Acceleration and curricular enrichment for gifted students: \\ a perspective of the situation in Peru
}

Jazmin Madrid Valdiviezo ${ }^{1}$

\begin{abstract}
RESUMEN
Se propone revisar teóricamente las principales estrategias de aceleración y enriquecimiento curricular dirigidas a estudiantes de alta capacidad. Se ha buscado información en el ámbito nacional e internacional y se han recopilado algunas estrategias que han demostrado ser útiles para el aprovechamiento del tiempo y mejora del talento académico. El tema en cuestión ha sido escasamente trabajado en el país, evidenciando falta de interés de los académicos, así como del sistema educativo peruano. Se enfatiza la importancia de que padres de familia y maestros puedan conocer y seleccionar alternativas efectivas para atender las necesidades de los estudiantes más capaces. Finalmente, se discute el tema desde una perspectiva psicoeducativa y de derechos, enmarcada en el contexto peruano.
\end{abstract}

\section{Palabras clave}

Estudiantes con altas capacidades, aceleración, enriquecimiento curricular.

\begin{abstract}
It is proposed to theoretically review the main strategies of acceleration and curricular enrichment aimed at gifted students. Information has been sought nationally and internationally and some strategies have been compiled that have demonstrated to be useful for time use and the enhancement of academic talent. The question at issue has been scarcely worked in the country, providing evidence lack of interest from academics, as well as the Peruvian education system. Emphasis is placed on the importance that parents and teachers being able to know and select effective alternatives to attend the needs of the most capable students. Finally, the subject is discussed from a psychoeducational and rights perspective, framed in the Peruvian context.
\end{abstract}

\section{Keywords}

Gifted students, acceleration, curricular enrichment.

\section{INTRODUCCIÓN}

La aceleración educativa de los estudiantes más capaces no ha tenido mucho apoyo de los docentes ni del sistema educativo peruano en general, como se evidencia en la escasa o nula información que se puede encontrar al respecto a través de los portales de Internet de instituciones públicas o privadas (por ejemplo,
Ministerio de Educación, Alicia-Concytec, etc.). Más allá de los Colegios de Alto Rendimiento que, según Piazzon, Boza, Palomino, Quijandría y Romero (2018) atienden a un $25 \%$ de esa población (al menos a aquellos procedentes de instituciones públicas), no se observa mayor interés político por atender a

1 Psicóloga, magíster en Ciencias de la Familia, experta en Altas Capacidades y Desarrollo del Talento (UNIR), y estudiante del doctorado en Psicología en la Universidad Femenina del Sagrado Corazón, UNIFÉ, Lima. jazmin.madrid@outlook.com 
estos estudiantes, probablemente porque se ha perpetuado el mito de que el estudiante de altas capacidades, por sus propios medios logrará desarrollarse, sin necesidad de apoyo externo (Rodrigues Maia-Pinto y de Souza, 2012), de ese modo mucho talento individual se pierde.

Esto no solo va en detrimento del propio individuo, sino que la falta de identificación y atención a los estudiantes con altas capacidades supone una pérdida de valioso capital humano que podría (y debería) contribuir significativamente al desarrollo del país con sus talentos y potencialidades. Tal como lo mencionan Piazzon et al. (2018), atender los requerimientos de los alumnos con mayor potencial de excelencia tiene una significativa relevancia social, por un lado, porque evidencia que se está brindando efectivamente atención a la diversidad, pero también porque mejora las condiciones sociales de las familias de estudiantes talentosos "lo que implica la reducción del índice de pobreza, ... reducción de la deserción escolar, con lo cual disminuyen (también) los niveles de delincuencia $y$ adicciones" (p. 47), convirtiéndose en el costo de oportunidad de atender a estudiantes con talento.

Como declara Tourón (2013) si no coexisten medidas deidentificación proactivas, periódicas y sistemáticas que permitan detectar el potencial de los estudiantes, así como indicadores con los cuales asegurar que los colegios atiendan oportunamente las necesidades educativas y de desarrollo curricular de las diferentes capacidades de sus estudiantes, entonces no se está trabajando para el desarrollo del talento. Ambos aspectos son fundamentales: la identificación y la atención. Es responsabilidad del Estado establecer políticas educativas que aseguren una mayor identificación y atención a los estudiantes talentosos de modo que se desarrolle su alto potencial social y económico, no como una política de un gobierno particular, sino como algo permanente que se sostenga a través del tiempo.

Los estudiantes de altas capacidades, por sus características deberían acceder a una educación individualizada y de acuerdo a su potencial. Sin embargo, muchas veces no se cuenta con el aval de una certera evaluación psicopedagógica, por lo que las posibilidades de acceder a una educación adecuada a su capacidad se hace menos viable; sumado a esto, el Ministerio de Educación (MINEDU, 2016) cuestiona la aceleración en los primeros años de escolaridad, probablemente por las dificultades que una incorrecta aplicación de esta haya generado sobre los mismos estudiantes y por las limitaciones logísticas y de personal para la identificación y diagnóstico. El problema es que, bajo el criterio de no matricularse en un grado superior a la edad, ningún estudiante podría acceder a ese tipo de aceleración educativa, y dado que la aceleración por definición implica "aprender al propio ritmo" o aprender de acuerdo a "las características de aprendizaje propias de cada individuo" (Reyero y Tourón, 2003, p. 113), la situación se torna más compleja. Frente a esta situación los padres de dichos niños, niñas y adolescentes - aquellos padres que sienten la necesidad de una educación inclusiva hacia los más capaces-, se encuentran desatendidos $y$ con pocas oportunidades que ofrecer a sus hijos.

Tomando en cuenta que los estudiantes con altas capacidades efectivamente son capaces de aprender más rápido, los programas educativos deberían responder a sus aptitudes intelectuales, por ejemplo, aumentando la velocidad de presentación, la profundidad y la amplitud de los contenidos en las diferentes materias. De ese modo la promoción escolar estaría "marcada por las capacidades demostradas de los alumnos y no por su edad" adecuando "el currículo al alumno y no el alumno al currículo" (Reyero y Tourón, 2003, p. 114). Sin embargo, siendo que las capacidades de los estudiantes, incluyen aspectos cognitivos, físicos, motivacionales y sociales, las prácticas acelerativas requieren de un estudio detallado, pertinente $e$ individualizado de cada estudiante de alta capacidad que lleve a decisiones que resulten satisfactorias para sus necesidades.

Al respecto, se propone esta revisión de estrategias acelerativas y de enriquecimiento curricular, con el propósito de despertar el 
interés de la comunidad académica y de los gestores educativos, así como responder modestamente- al interés de los padres de familia, de manera que se puedan identificar algunas alternativas educativas para estudiantes de altas capacidades, priorizándose el análisis de opciones de intervención y su respectivo contraste con lo que sucede en la educación peruana actual.

\section{Aceleración y enriquecimiento curricular}

De acuerdo con Reyero y Tourón (2003) las dos principales alternativas educativas que suelen emplearse con estudiantes de alta capacidad son la aceleración y el enriquecimiento curricular. A continuación, se plantean algunas definiciones, así como las principales estrategias que se derivan de estos enfoques.

Existen muchas definiciones de aceleración educativa, algunas definiciones inciden en la edad y en el progreso en años escolares, como por ejemplo el informe Templeton que puntualiza que la aceleración es "una intervención educativa que hace avanzar a los estudiantes a través de un programa educativo, a un ritmo más acelerado que el usual o a una edad menor a la típica" (Colangelo, Assouline y Gross, 2004, p. 5). Otras, como la de Benbow (1998, citada por Reyero y Tourón, 2003), quien proponen que "la aceleración consiste ... en decidir que la competencia, más que la edad, debería ser el criterio para determinar cuándo un alumno está preparado para acceder a un determinado currículo o a unas determinadas experiencias académicas" (p. 115), en este caso, el énfasis es puesto en las habilidades demostradas. También Reyero y Tourón (2003) consideran que la aceleración

es sinónimo de aprender al propio ritmo, lo que implica saber combinar adecuadamente sus tres ingredientes principales: profundidad, velocidad $\mathrm{y}$ amplitud, así como tener en cuenta las características cognitivas, físicas, sociales y emocionales de cada uno de los alumnos susceptibles de ser acelerados. (p. 114)
En lo que concierne al enriquecimiento curricular Reyero y Tourón (2003) explican que las experiencias de aprendizaje de los estudiantes son ampliadas y profundizadas mediante la implementación de estrategias que no suelen estar en el currículo, diferenciándose de la aceleración en que no avanza más rápidamente a través del currículo. Entre sus ventajas están: la profundización de los temas de estudio; que no se separa al estudiante talentoso de sus compañeros de edad preservándolo de ser afectado social o emocionalmente; que permite profundizar en temas de interés para el estudiante incluso si no están en el currículo; que genera menos dificultades a los administradores de los colegios, entre otras. Cabe resaltar que la clave para un buen aprovechamiento del enriquecimiento está en un plan curricular sistemático, es decir, el diseño debe ser preciso, intencionado y consciente de las necesidades específicas del estudiante dotado (Wu, 2016). No obstante, Stanley (1976) quien clasifica al enriquecimiento en cuatro tipos: el enriquecimiento de "trabajo arduo", el académico irrelevante, el cultural y el académico relevante (Tabla 1), estima que cuanto más destacado sea el enriquecimiento, mayor será el llamado a acelerar la asignatura posteriormente. De lo contrario, solo se inhibe el aburrimiento, pero prácticamente se garantiza que con el tiempo este sea más severo que nunca, tendiendo así a favorecer la aceleración sobre el enriquecimiento. 
Tabla 1

Tipos de enriquecimiento curricular

Enriquecimiento de "tra- El estudiante se mantiene en el mismo nivel, solo incrementa el trabajo arduo" bajo académico en comparación con el resto de sus compañeros de aula.

Enriquecimiento acadé- $\quad$ El estudiante se mantiene en el mismo nivel, solo incrementa el tramico irrelevante bajo académico en comparación con el resto de sus compañeros de aula.

Enriquecimiento cultural $\quad$ El enriquecimiento cultural pretende proporcionar desarrollos en artes escénicas, música, artes visuales, danza, escritura creativa, etc., pero nuevamente no se atienden las necesidades específicas de aquellos con talentos intelectuales especiales. Corresponde aplicar este tipo de enriquecimiento si el estudiante posee talentos para alguna de las artes.

Enriquecimiento acadé- La clave está en que lo que se ofrece al estudiante sea realmente mico relevante relevante para el desarrollo de sus talentos específicos, es decir, darle un tratamiento relacionado con sus aptitudes especiales y en un grado más elevado.

Fuente: Adaptado de Stanley (1976).

\section{Estrategias de aceleración y enriquecimiento curricular}

En el Informe Templeton se mencionan 18 tipos de aceleración (Colangelo, et al., 2004); a continuación, en la Tabla 2 se describe cada tipo al mismo tiempo que se reflexiona sobre lo que sucede en el entorno peruano. Por su parte, Davis, Rimm y Siegle (2014) listan distintos programas de enriquecimiento (Tabla 3), a los que también se les dará una mirada contextualizada.

Tabla 2

Tipos de aceleración

Admisión temprana al Según lo establecido por la política del estado peruano, se requiere nivel inicial haber cumplido al 31 de marzo la edad de 3, 4 o 5 años, según sea el caso. Si se permitiera la admisión temprana, los niños podrían ingresar al nivel inicial antes de alcanzar la edad mínima requerida.

Admisión temprana a la Igual al anterior, considerando que la primaria se inicia con 6 años primaria cumplidos, si se practicara este tipo de aceleración se podría obviar el nivel inicial o acelerar a un estudiante de inicial al primer grado de primaria o más.

Se considera que un estudiante ha omitido la calificación si se lo Omisión de calificación asigna a un grado por delante de sus compañeros en edad cronolóy/o saltarse grados gica. El salto de grado se puede hacer al principio o durante el año escolar.

Progreso continuo El estudiante recibe contenido poco a poco, en la medida que domina el contenido anterior. Cuando su progreso supera el desempeño de sus compañeros en edad, entonces se acelera y pasa al siguiente nivel. 
Instrucción al propio ritmo

El estudiante selecciona por sí mismo las actividades de instrucción que considera más adecuadas. Es similar al anterior: un subtipo de aceleración de progreso continuo. La diferencia está en que el estudiante decide lo que desea aprender.

Aceleración de asignaturas o aceleración parcial

En este caso, el estudiante participa de algunas clases con compañeros mayores al menos algunas horas o con materiales de un nivel superior en algunas asignaturas. También se puede realizar en otros momentos (por ejemplo, en verano, por las tardes o fines de semana) pero sin dejar a sus compañeros de edad, en todas las demás asignaturas.

Clases combinadas $\quad$ Aunque no es, en sí misma, una práctica diseñada para la aceleración, en algunos casos compartir el aula con compañeros de grados superiores, permite a los estudiantes menores relacionarse académica y socialmente con ellos, lo que podría resultar en una colocación de grado avanzado más tarde.

Compactación del plan Consiste en reducir la cantidad de actividades introductorias, ejercide estudios cios y práctica, incluso disminuir los objetivos de instrucción del plan general de estudios. Al ganarse tiempo de este modo, se puede plantear contenido más avanzado o enriquecido. Los objetivos educativos deben seleccionarse cuidadosamente en contenido y respetarse la jerarquía del currículo.

Currículo compacto El estudiante recibe instrucción que implica menos tiempo de lo normal (por ejemplo, completar en un semestre todo un grado; o hacer en dos años, lo que corresponde a tres grados de secundaria). Se diferencia del anterior, en que el tiempo ahorrado permite que se avance de grado.

Tutoría Se refiere a contar con un mentor o un tutor experto, que proporcio-
na oportunidades de aprendizaje acelerados.

Programas extracurriculares El estudiante elige cursos para realizar al terminar sus clases o en el verano, que confieren créditos académicos o temas de nivel superior.

\begin{tabular}{ll}
\hline Cursos en línea & $\begin{array}{l}\text { El estudiante se inscribe en cursos impartidos por Internet, lo que le } \\
\text { permite adelantar en ciertos temas de su interés. }\end{array}$ \\
\hline
\end{tabular}
Graduación temprana Se alcanza cuando el estudiante es capaz de graduarse de la escuela secundaria o de la universidad en tres años y medio o menos. Esto suele lograrse cuando se aumentan los cursos realizados cada año, a través de la inscripción doble, de cursos extracurriculares o inscribiéndose en cursos por internet.

Inscripción simultánea o $\quad$ El estudiante aprueba una asignatura en un nivel (por ejemplo, en la doble secundaria) y al mismo tiempo, recibe crédito por un curso similar en un nivel superior (por ejemplo, en la universidad).

Colocación avanzada $\quad$ El estudiante toma un curso que otorgará créditos universitarios al cumplir exitosamente una prueba normalizada, un ejemplo de esto, son los créditos académicos universitarios que ganan los estudiantes que aprueban el Programa de Diploma de la Organización del Bachillerato Internacional.

Créditos por examen $\quad$ El estudiante obtiene créditos académicos avanzados al completar con éxito algún examen o alguna otra evidencia de dominio. 
Aceleración en la universidad

Ingreso temprano a la secundaria o universidad
El estudiante adelanta al menos un año de estudios universitarios. Se puede conseguir al fusionar técnicas de aceleración, como la inscripción doble, el crédito por examen o si los maestros y administradores universitarios así lo determinan.

El estudiante empieza la universidad sin haber concluido cada grado de la secundaria. También es el caso del estudiante universitario que concluye dos carreras universitarias por el mismo tiempo u obtiene un grado avanzado (por ejemplo, maestro) junto con el título universitario. En el Perú, algunos estudiantes de secundaria pueden postular e incluso algunos ingresan con puestos destacados a la universidad, pero no se tiene conocimiento de que se les permita estudiar si no han concluido la secundaria formalmente.

Instituciones especializadas en estudiantes talentosos (agrupamiento por capacidad)

El estudiante asiste a una institución educativa que ofrece un programa de estudios más amplio y profundo para cada uno de los grados. Un ejemplo de esto podrían ser los Colegios de Alto Rendimiento o los Programa de atención Educativa para Niños con facultades Talentosas Sobresalientes (PAENFTS).

Fuente: Adaptado de Colangelo, Assouline y Gross (2004).

\section{Tabla 3}

Estrategias de enriquecimiento

Proyectos de investiga- Un proyecto de investigación en biblioteca o en Internet debe basarción en bibliotecas e internet se en un fuerte interés, habilidad para el estudio autónomo e iniciativa por parte de los estudiantes. Como cualquier proyecto independiente, es importante que se logre un producto y se exponga ante el público puesto que la calidad del trabajo de los estudiantes aumenta exponencialmente cuando se presenta a una audiencia real.

Proyectos de investiga- La respuesta a un problema de investigación auténtico no es algo ción científica que se pueda encontrar en un libro o en un sitio web, requiere descubrir mediante un proceso de recopilación y análisis de información. La investigación de esta naturaleza se basa en el interés de los estudiantes e implica que estos desarrollen una variedad de habilidades de investigación, resolución de problemas y pensamiento creativo. Demanda el acompañamiento de un profesor-asesor.

Proyectos independientes Los estudiantes elaboran proyectos de arte de su interés (por ej. dien arte, drama, escritura bujo, pintura, escultura, serigrafía, letras, cerámica, fotografía o tejicreativa y otros do, entre otros), aprenden el proceso de elaborar un producto creativo y se generan oportunidades para que sea publicado o presentado en algún evento o concurso.

Centros de aprendizaje Son ambientes creados especialmente para favorecer el aprendizaje de los estudiantes dotados, por ejemplo, una biblioteca equipada con materiales de lectura y juegos educativos; computadoras para la escritura creativa. O un centro de matemáticas con kits y juegos matemáticos, calculadoras, sistemas computarizados con juegos de lógica, desafíos matemáticos, etc.

Excursiones Los viajes de campo se pueden utilizar como una actividad exploratoria dirigida a familiarizar a los estudiantes con áreas culturales o científicas o con posibilidades de carrera. Las excursiones también 
pueden ser una fuente de información para los proyectos independientes de los estudiantes.

Las excursiones son más beneficiosas si los estudiantes tienen problemas específicos para resolver, preguntas para responder o proyectos o presentaciones posteriores a la gira para prepararse.

Programas de fin de semana o para después de la escuela
Estos programas permiten que los estudiantes dotados se encuentren y trabajen juntos lejos del estrés y los problemas de los requisitos escolares diarios. Suelen ser miniclases dirigidas por maestros voluntarios, profesores universitarios, estudiantes graduados o expertos de la comunidad, incluso, padres de niños con alta capacidad. Estos voluntarios suelen estar comprometidos y se sienten entusiasmados de trabajar con estudiantes dotados.

Programas de verano En verano se pueden aprovechar las vacaciones usualmente más largas para promover actividades de aprendizaje enriquecido. Al participar de programas especializados se pueden graduar mejor los niveles de dificultad y ritmo, combatiendo así los malos hábitos de estudio y el bajo rendimiento de las clases demasiado fáciles y aburridas; por ejemplo, en programas residenciales, se desarrolla la independencia y habilidades de vida, entre otras ventajas.

Programas de mentoría Los beneficios de la mentoría de estudiantes dotados, trascienden las ventajas académicas y profesionales y, por lo general, incorporan una mayor confianza en sí mismos, responsabilidad y propósito. Cuando se cuenta con mentores estos reconocen las características, necesidades y problemas de cada estudiante a su cargo y apoyan siendo modelos positivos según sea necesario.

Fuente: Adaptado de Davis, Rimm y Siegle (2014).

\section{Método}

Para esta revisión se utilizó el buscador Google, con localización en el Perú y con las palabras clave en español: aceleración educativa en el Perú, enriquecimiento curricular en el Perú y otras similares, encontrándose algunos datos en informes, artículos periodísticos y páginas informativas. También se realizaron búsquedas con términos semejantes en el repositorio digital nacional Alicia que gestiona los repositorios de universidades peruanas y de otras entidades públicas y privadas, sin mucho éxito. Del mismo modo se revisaron artículos en español $e$ inglés de contextos extranjeros, relevantes para fundamentar teóricamente esta revisión. Cabe resaltar, que es escasa la información que se ha encontrado sobre lo que sucede en el Perú respecto a aceleración y enriquecimiento curricular, probablemente porque la atención a estudiantes talentosos académicamente no ha sido un tema prioritario para el país y también porque mucho de lo que se hace en las instituciones educativas, no suele evidenciarse mediante publicaciones académicas o informativas.

\section{Discusión}

A lo largo de los años, la aceleración educativa ha generado mucho debate, quienes están a favor destacan todas las ventajas de adelantar grados y quienes están en contra, cuestionan los efectos socioemocionales que, al no estar en su grupo etario, podría tener sobre el estudiante, incluso algunos la contraponen al enriquecimiento curricular, aunque son estrategias diferentes (Reyero y Tourón, 2003). Esta discusión, está lejos de resolverse.

Partiendo de una mirada psicopedagógica, tanto las estrategias de aceleración como las de enriquecimiento pueden beneficiar al estudiante, la clave en la elección de la o las estrategias está en una completa y adecuada evaluación no solo a nivel individual, sino familiar y social, de modo -que se puedan plantear a 
los padres y estudiante estrategias relevantes a la naturaleza específica de los talentos que este posee (Stanley, 1976). En consecuencia, es responsabilidad de los profesionales psicopedagogos, en primer lugar, capacitarse para una adecuada identificación, evaluación y orientación a los estudiantes más capaces y a sus familias; en segundo lugar, ya en el campo mismo de la orientación, ofrecer estrategias acorde a las necesidades de los más capaces esclareciendo sus ventajas y desventajas, así, los padres de familia junto con el estudiante podrán tomar decisiones informadas, de acuerdo a su perfil y posibilidades.

En esa línea, es importante que el orientador psicopedagógico pueda discernir y ayudar a diferenciar, entre un enriquecimiento relevante para ese estudiante en particular de uno irrelevante, el énfasis está en "considerar la naturaleza específica de los talentos del estudiante", cuidando de que no se ofrezcan actividades que impliquen únicamente "trabajo arduo" sin sentido o importancia para el mismo estudiante $\mathrm{u}$ ofreciendo un enriquecimiento cultural, que si bien es positivo, tal vez no responda a sus demandas intelectuales.

Cabe destacar la importancia de respetar la normativa educativa peruana, respecto a la aceleración o no de los estudiantes, en estos casos el enriquecimiento curricular en sus diferentes modalidades puede ser la opción que más se ajuste. A muchos padres les gustaría que sus hijos sean acelerados algunos grados, sin embargo, no es la única estrategia ni la más efectiva; cada caso es único, y debe evaluarse la pertinencia de la aceleración cuidadosamente.

Desde una perspectiva de derechos, si bien la Ley General de Educación 28044 (2003), reconoce que los niños y adolescentes talentosos "son sujetos directos de la acción de educación básica especial y que su educación se debe impartir con miras a su inclusión en aulas regulares, sin perjuicio de la atención complementaria y personalizada que requieran" (Art. 39), también es cierto que llevar a la práctica esto resulta una tarea ardua y compleja. En una relectura de este derecho de los estudiantes de altas capacidades, tal como está escrito, podría estar referido al diseño de actividades de enri- quecimiento más que a considerar alguna propuesta acelerativa.

Cabe destacar que la experiencia de los Colegios de Alto Rendimiento es una respuesta al compromiso asumido por el Estado de ofrecer programas educativos especializados con el fin de lograr el desarrollo de las potencialidades de los estudiantes con alta capacidad (Ley 28044, 2003, Art. 18, inciso f). Así, el convocar a estudiantes de primeros puestos de instituciones educativas públicas, puede ser considerada una estrategia de agrupamiento, no obstante, en sí misma no implica aceleración, pues los estudiantes deberán estudiar tercero, cuarto y quinto de secundaria, según corresponda a su edad. Una vez dentro, acceden al Programa del Diploma del Bachillerato Internacional (OBI) el cual está dirigido regularmente a estudiantes de entre 16 y 19 años, empero, en el Perú se destina a estudiantes que cursan cuarto y quinto de secundaria (de 15 y 16 años), planteado de ese modo se puede decir que involucra cierta aceleración, si bien lo más desafiante está en participar de un currículo de alto nivel de exigencia académica. También el estudiante tiene la posibilidad de acumular créditos académicos en algunas asignaturas de estudios generales, susceptibles de ser convalidadas en algunas universidades nacionales y extranjeras - algo que no se consigue fácilmente-, pues requiere esfuerzo y dedicación para aprobar una serie de exámenes estandarizados internacionalmente.

Por otro lado, el Programa Nacional de Becas y Crédito Educativo, desde el año 2012 implementa Beca 18 aumentando significativamente el acceso a becas y ayudas para acceder a una educación de nivel terciario. Entre sus requisitos pide contar con alto rendimiento académico y está dirigido especialmente a aquellos que no cuentan con recursos económicos para cubrir los costos de su educación (Ley 28044, Art. 18, inciso $\mathrm{H}$ ), es así que estudiantes de alta capacidad, sin importar si su condición económica es baja podrían acceder a estas oportunidades, compitiendo por una vacante, en igualdad de condiciones, con otros estudiantes.

Una experiencia de aceleración, aunque no ha sido estrictamente para este grupo, muestra 
la posibilidad de implementar proyectos que ayuden a estos estudiantes a avanzar académicamente. Por ejemplo, está el piloto de Aceleración $2 \times 1$ que en la región Junín ha beneficiado a 1338 estudiantes de 2do a 5to grado de primaria, y sirvió para reducir el rezago escolar de niños afectados por el trabajo infantil (Vivanco, 2017); por otro lado, el Ministerio de Educación viene implementando programas de atención a población rural con rezago escolar (por ejemplo, Secundaria Tutorial), que hace pensar en que la atención a la diversidad no solo debería darse en el extremo rezagado, sino que por equidad, también debería ofrecerse oportunidades de aceleración a estudiantes con alta capacidad. Estas experiencias revelan que con voluntad política se puede llegar a atender a poblaciones con necesidades específicas de educación.

Evaluar, como país, el estado de esta cuestión, sería un importante paso al respecto (ver, por ejemplo, los informes State of the Nation in Gifted Education 2010-2011 y 2014-2015 realizado por la National Association for Gifted Children (2016) y el Council of State Directors of Programs for the Gifted). Sin embargo, este tipo de monitoreo, seguimiento y evaluación no ocurre en el Perú, y en general, tampoco en la región latinoamericana, al menos no de un modo sistemático. Tourón (2016) expresa que la investigación empírica a favor de la aceleración es cada vez más numerosa (al menos en EEUU y países europeos), "revisiones y meta-análisis han mostrado que la aceleración funciona tanto a corto como a largo plazo, y ŚmanifiestaȘ que los supuestos efectos negativos que afectan al desarrollo social y emocional de los niños no son reales" (párr. 6). Sin embargo, en nuestro país se carece de investigación teórica y empírica ajustada a este contexto que valide tales aseveraciones. Al respecto, se suele importar resultados de investigaciones realizadas en otros países que, por lo general, no se ciñen a nuestra particular realidad, mostrando que en cuestión de altas capacidades y aceleración todavía hay mucho que estudiar y demostrarse.

\section{Conclusiones}

En conclusión, la atención a la diversidad, enfocada en la aceleración educativa de los más capaces, requiere mayor atención por parte de todos los actores del sistema educativo peruano, en especial, los que lideran las políticas educativas. En ese sentido, se sugiere ampliar el marco normativo para la atención de los estudiantes talentosos, que incluya a todos los niveles educativos tanto de gestión estatal como no estatal; que promueva la formación de profesionales (psicólogos, docentes, etc.) especializados en identificación, evaluación y orientación de niños, adolescentes y adultos con altas capacidades; que proponga protocolos de evaluación y atención; que lleve a cabo procesos de identificación sistemáticos y periódicos a nivel local y nacional; que monitoree y haga seguimiento a las acciones de detección y atención del talento que realicen las instituciones educativas; entre otros.

Debe romperse con la inercia y con ese mito que está profundamente arraigado: que los estudiantes más capaces no precisan apoyo externo, todo lo contrario, lo necesitan y mucho. Pero no es solo apoyo económico u oportunidades para adelantar académicamente, también se necesita desarrollar en ellos, conciencia ciudadana y vocación de servicio para que, cualquiera sea el camino que elijan para desplegar su liderazgo y talentos individuales, sean capaces de contribuir desde su espacio de influencia al desarrollo del Perú. 


\section{REFERENCIAS}

Colangelo, N., Assouline, S., Gross, M. (Eds.) (2004). Una nación engañada: De quéforma las escuelas reprimen a los estudiantes más brillantes de los EEUU. The Connie Belin \& Jacqueline N. Blank International Center for Gifted Education and Talent Development. (Informe nacional Templeton). Recuperado de http://www.accelerationinstitute.org/nation_deceived/International/ND_v1_es.pdf

Davis, G. A., Rimm, S. B. \& Siegle, D. (2014). Education of the gifted and talented (6th ed.). Boston: Pearson Education.

Ley 28044. Ley General de Educación. Congreso de la República del Perú. (2003). Recuperado de http://www.minedu.gob.pe/p/ley_general_de_educacion_28044.pdf

Ministerio de Educación (2016). Plan de gestión institucional del programa nacional de becas y crédito educativo del ministerio de educación, 2012 - 2016. Recuperado de http://www.pronabec. gob.pe/inicio/publicaciones/documentos/pgi_2016.pdf

National Association for Gifted Children (2016). State of the nation in gifted education. Recuperado de http://www.nagc.org/sites/default/files/key\%20reports/2014-2015\%20State\%20of\%20 the\%20States\%20summary.pdf

Piazzon, L., Boza, D., Palomino, E., Quijandría, T. y Romero, K. (2018). Factores críticos de éxito para la incorporación y permanencia de las instituciones educativas públicas de Lima en el Sistema de Bachillerato Internacional. Lima: Esan ediciones. Recuperado de http://repositorio.esan. edu.pe/bitstream/handle/ESAN/1224/SGD_70.pdf?sequence $=1 \&$ isAllowed =y

Rodrigues Maia-Pinto, R. y de Souza Fleith, D. (2012). Aceleración de la enseñanza para alumnos superdotados: argumentos favorables y contrarios. Revista de Psicología [PUCP], 30(1), 189-214. Recuperado de http://www.scielo.org.pe/scielo.php?script=sci_arttext\&pi$\mathrm{d}=$ S0254-92472012000100008\&lng=es\&tlng=es.

Reyero, M. y Tourón, J. (2003). El desarrollo del talento y la aceleración como estrategia educativa. La Coruña: Netbiblo.

Stanley, J. C. (1976). The case for extreme educational acceleration of intellectually brilliant youths. Gifted Child Quarterly, 20(1), 66-75.

Tourón, J. (2013). iEl Estado de la Nación: o de cómo tomarse en serio el desarrollo del talento! [Mensaje en un blog]. Recuperado de https://www.javiertouron.es/el-estado-de-la-nacion-o-de-como/

Tourón, J. (2016). La aceleración: capacidad frente a edad I (8). Recuperado de https://www. javiertouron.es/la-aceleracion-capacidad-frente-edad-i-8/

Vivanco, T. (25 de febrero de 2017). Programa Aceleración 2x1 busca reducir la cifra de rezago escolar. Diario Correo. Recuperado de https://diariocorreo.pe/edicion/huancayo/programa-aceleracion-2x1-busca-reducir-la-cifra-de-rezago-escolar-733515/

Wu, E. (2013). Enrichment and acceleration: Best practice for the gifted and talented. Gifted Education Press Quarterly, 27(2). Recuperado de https://www.researchgate.net/publication/311831051_Enrichment_and_Acceleration_Best_Practice_for_the_Gifted_and_Talented

Fecha de recepción: 03-11-18

Fecha de aceptación: 18-11-18. 